\title{
Síntese e caracterização de materiais do tipo MCM-41sulfatada
}

Kleberson Ricardo de Oliveira Pereira* (Pesquisador na Unidade Acadêmica de Engenharia de Materiais,

Universidade Federal de Campina Grande - UFCG).

Adalício Uzêda Antunes Júnior (Mestrando em Engenharia Química, Universidade Federal de Campina Grande - UFCG).

Maria Wilma Nunes Cordeiro Carvalho (Prof. a Titular da Unidade Acadêmica de Engenharia Química,

Universidade Federal de Campina Grande - UFCG).

Adriano Sant’Ana Silva (Prof. da Unidade Acadêmica de Tecnologia de Alimentos, Universidade

Federal de Campina Grande - UFCG).

*Email: klebersonric@gmail.com

Resumo:

Os processos químicos consistem na transformação de matérias primas em produtos por meio de reações químicas. Reações em processos industriais necessitam ter elevada eficiência, rápido processamento e boa qualidade, o que pode ser alcançado através do uso de catalisadores. Devido aos problemas ambientais, a catálise mostra-se como alternativa promissora em promover vias sustentáveis de produção e, configura-se como fundamental para a melhoria de processos químicos.

De forma geral, as reações catalíticas podem ser classificadas em homogêneas e heterogêneas. $\mathrm{O}$ uso de catalisadores heterogêneos ganha destaque devido à possibilidade de redução de impactos ambientais e aumento da eficiência de processos. Visando a substituição de catalisadores homogêneos, o emprego de catalisadores sólidos vem sendo difundida cada vez mais no meio científico. Catalisadores heterogêneos mesoporosos, como Mobil Compositionof Matter-41 (MCM-41), vem sendo estudados por diversos pesquisadores na aplicação em reações envolvendo macromoléculas. As principais vantagens do uso dessa peneira molecular mesoporosa são: elevada área superficial, elevado diâmetro de poro e volume poroso e boa estabilidade térmica.

A MCM-41 é um material inativo cataliticamente ou pode apresentar sítios ácidos muito fracos, estes materiais mesoporosos, por sua vez podem ter sua superfície modificada mediante a incorporação de uma fase ativa. Dentre as quais se destacam a adição de heteroátomos para geração de sítios ácidos e o íon sulfato o qual confere ao material a característica de superácido. Diante disso, o objetivo deste estudo foi avaliar a síntese e caracterização do suporte MCM-41 e dos catalisadores $\mathrm{ZrO}_{2}-\mathrm{MCM}-41$ e $\mathrm{SO}_{4}{ }^{2-}-\mathrm{ZrO}_{2}-\mathrm{MCM}-41$. Os catalisadores foram sintetizados pelo método de incorporação por mistura física do óxido com concentração de $5 \%$ de $\mathrm{ZrO}_{2}$ em relação mássica e em seguida caracterizados por DRX, EDX e MEV.

Os resultados sugerem que o comportamento característico do material mesoporoso MCM41 foi alcançado após as sínteses e que os materiais são constituídos basicamente por sílica. Alternativamente, observou-se a presença de compostos derivados de zircônia e enxofre após análise dos catalisadores. Em relação à morfologia, verificou-se que os materiais apresentam formas heterogêneas com presença de aglomerados.

Palavras-chave: Peneiras moleculares; MCM-41; Adsorção; Catálise Heterogênea. 


\section{I ntrodução}

A maioria das sínteses industriais e quase todas as reações biológicas requerem catalisadores. Estima-se que cerca de $90 \%$ dos produtos químicos sejam obtidos mediante processos que requerem o uso de um catalisador em, pelo menos, uma das etapas de sua produção (ARMOR, 2011). Tal fato ressalta a importância do estudo da catálise e aplicação em diversos setores.

De forma geral, as reações catalíticas podem ser classificadas em homogêneas, se os catalisadores estão presentes na mesma fase dos reagentes, ou, heterogêneas, em que os catalisadores estão presentes em fase diferente daquela dos reagentes (SCHMAL, 2011).

Para a indústria, o emprego de catalisadores homogêneos acarreta diversos problemas técnicos e ambientais, como corrosão, formação de rejeitos e separação dos produtos obtidos, do catalisador e dos solventes utilizados (VÉDRINE, 2014).

Os problemas citados anteriormente são minimizados com o uso de catalisadores heterogêneos, que facilitam a separação dos produtos e, em muitos casos, podem ser regenerados e reutilizados, provocam pouca ou nenhuma corrosão, são de fácil manuseio e possibilitam o fácil reinício de processos contínuos em reações de leito fixo. Eles apresentam alta estabilidade térmica, elevada atividade catalítica e seletividade perante vários tipos de reação (ZHANG et al., 2010).

Materiais mesoporosos e macroporosos são selecionados para os processos catalíticos que envolvem moléculas de grandes dimensões e que permitem a difusão do líquido para os centros ativos no interior de canais regulares internos (SU e GUO, 2014).

Como característica bastante vantajosa relacionada às peneiras moleculares mesoporosas, tem-se o fato delas poderem servir como suportes e/ou catalisadores para aplicações catalíticas (YANG et al., 2014).

Dentre as peneiras moleculares mesoporosas, a MCM-41 ganhou destaque devido, principalmente as seguintes características: poros ordenados e uniformes com diâmetro de poro variável de 2 a $10 \mathrm{~nm}$; elevada área superficial com valores superiores a $800 \mathrm{~m}^{2} \mathrm{~g}^{-1}$; grande capacidade de adsorção; acessibilidade de moléculas grandes aos sítios ativos no interior dos poros, entre outros (CASTRO, 2009).

Peneiras moleculares, como a MCM-41, constituídas de estrutura puramente de silício, que são quimicamente inertes (carga elétrica nula) e assim ocasionam baixa força ácida, o que exige a introdução de uma fase ativa, para que venha apresentar propriedades ácidas ou básicas (Lewis ou Brönsted) (CHEN et al., 2014).

O estudo da acidez nos materiais catalíticos é importante na medida em que a determinação da força dos sítios ácidos existentes na superfície do catalisador e também a distribuição, se apresenta como condição fundamental para análise da atividade e da seletividade do catalisador, relacionando estas análises com as propriedades ácidas.

Os sítios ácidos presentes na superfície dos catalisadores heterogêneos podem ser classificados em sítios ácidos de Brönsted e sítios ácidos de Lewis. Os sítios ácidos de Brönsted, também chamados centros protônicos, são espécies químicas capazes de atuar como doadora do cátion hidrogênio (próton, $\mathrm{H}^{+}$). A acidez de Lewis está comumente associada aos sistemas não próticos resultantes da interação com metais, principalmente os metais de transição por meio de seus orbitais d incompletos, capazes de receber elétrons. Frequentemente, estes metais formam catalisadores heterogêneos com a habilidade de processar com eficiência diversas reações químicas (MORENO e RAJAGOPAL, 2009).

A incorporação do ânion sulfato $\left(\mathrm{SO}_{4}{ }^{2-}\right)$ a este suporte permite aumentar a atividade catalítica deste material devido à formação de sítios catalíticos conhecidos como superácidos. Um sólido superácido é definido como um material sólido que apresenta uma força ácida maior que a força ácida de ácido sulfúrico 100\%, ou seja, pka $\leq-12$ (TANABE e HÖLDERICH, 1999).

A super acidez desses materiais é atribuída à formação de vários sítios ácidos de Brönsted que são vizinhos a poderosos sítios ácidos de Lewis e ambos os sítios tendem a aumentar a acidez desse catalisador. O sítio ácido de Lewis aparece devido ao efeito indutor exercido pelo ânion sulfato em relação ao íon metálico que fica deficiente de elétrons. Por outro lado, os sítios ácidos de Brönsted são formados pela presença de água na estrutura do material (ONDA et al., 2009). 
A zircônia sulfatada é um catalisador ácido forte, e outros catalisadores ácidos sólidos típicos, como as zeólitas, não apresentam atividade equivalente. Um dos fatores que controlam a atividade catalítica da zircônia sulfatada é a quantidade de íons sulfato na sua superfície (PARVULESCU et al., 1998).

Tipicamente, a área superficial da zircônia sulfatada está em torno de $100 \mathrm{~m}^{2} \mathrm{~g}^{-1} \mathrm{em}$ uma fase cristalina tetragonal. Entretanto, pela sua baixa área superficial e pequeno diâmetro de poros, seu uso pode ser muito limitado na hidrólise da celulose, em virtude da dimensão desta molécula (CORMA et al., 1997). Contudo, por sua elevada estabilidade térmica, área superficial específica e diâmetro de poros, a peneira molecular mesoporosa MCM-41 destaca-se como excelente suporte para a zircônia sulfatada, apresentando assim atividade e seletividade superiores ao uso de sílica amorfa, alumina e zeólitas.

Diante disso, o intuito deste trabalho é avaliar as propriedades químicas de peneiras mesoporosas obtidas a partir da MCM-41 e verificar as etapas de incorporação de óxido de zircônio e sulfatação na estrutura do material.

\section{Materiais e métodos}

\subsection{Síntese da MCM-41}

Os reagentes utilizados na síntese foram o brometo de cetiltrimetilamônio (CTMABr) (97\%, Merck), tetraetilortosilicato (TEOS) (98\%, Merck), amônia em solução (25\%, Merck) e água deionizada.

O suporte mesoporoso MCM-41 foi sintetizado de acordo com metodologia descrita por Gaydhankar et al. (2007). O processo consistiu na dissolução, sob agitação constante, de $\mathrm{CTMABr}$ (direcionador) em água deionizada. Em seguida, adicionou-se a solução de amônia à mistura, a qual permaneceu sob agitação por 10 minutos. Finalizando o processo, foi acrescentado, gota a gota, com auxílio de bureta, o TEOS. O gel de síntese foi mantido sob agitação constante por 4 horas a temperatura ambiente. A composição molar do gel de síntese foi $\mathrm{SiO}_{2} / 0,12 \mathrm{CTABr} / 2,50 \mathrm{NH}_{4} \mathrm{OH} / 150 \mathrm{H}_{2} \mathrm{O}$.

Após este período o gel foi filtrado sob vácuo e o material obtido lavado com água deionizada (cerca de $500 \mathrm{~mL}$ ). O filtrado obtido foi encaminhado para secagem em estufa, sem circulação de ar, a $70^{\circ} \mathrm{C}$ por 24 horas. E após seco foi realizada a etapa de calcinação, para a retirada do direcionador, realizada em forno tipo mufla a $550^{\circ} \mathrm{C}$.

\subsection{I ncorporação do óxido de zircônio e sulfatação}

A incorporação do óxido de zircônio $\left(\mathrm{ZrO}_{2}\right)$ foi realizada de acordo com procedimento descrito por Sun et al. (2002), em que o oxicloreto de zircônio octahidratado $\left(\mathrm{ZrOCl}_{2} \cdot 8 \mathrm{H}_{2} \mathrm{O}\right)$ foi misturado, em processo por batelada, a 2 gramas de MCM-41 mediante mistura física por 20 minutos e por seguinte seco em estufa a $70^{\circ} \mathrm{C}$ por 12 horas e, posteriormente, a $140^{\circ} \mathrm{C}$ por 4 horas. $\mathrm{O} \mathrm{ZrO}_{2}$ foi introduzido na concentração de $5 \%$ em relação a massa do suporte.

Uma vez introduzido o $\mathrm{ZrO}_{2}$, o ânion sulfato $\left(\mathrm{SO}_{4}{ }^{2-}\right)$ foi impregnado ao material $\mathrm{ZrO}_{2}$ MCM-41, por meio do método de impregnação por via úmida, tendo uma solução de ácido sulfúrico como fonte precursora do ânion (AQUINO et al., 2001). O processo consistiu na adição de 2 gramas a $60 \mathrm{~mL}$ de uma solução de ácido sulfúrico $\left(40 \mathrm{mmol} \mathrm{L}^{-1}\right)$. Posteriormente, a mistura foi agitada por 2 horas em agitador magnético e então foi disposta em estufa para total evaporação da fase líquida a $65^{\circ} \mathrm{C}$. Uma vez evaporada a fase líquida, o material foi exposto ainda a $110^{\circ} \mathrm{C}$ por 6 horas. Depois de seca em estufa a amostra $\mathrm{SO}_{4}{ }^{2-}-\mathrm{ZrO}_{2}-\mathrm{MCM}-41$ foi calcinada a $600^{\circ} \mathrm{C} \mathrm{em}$ atmosfera de nitrogênio por 3 horas. 


\subsection{Caracterizações}

O suporte MCM-41 e os catalisadores foram caracterizados por difração de raios X (DRX), fluorescência de raios X por energia dispersiva (EDX) e microscopia eletrônica de varredura (MEV).

Em relação à análise por DRX, os ensaios foram realizados em difratômetro Shimadzu, modelo XRD 6000, com fonte de radiação $\operatorname{CuK} \alpha(\lambda=1,5406 \AA)$, obtida por $40 \mathrm{kV}$ em corrente de filamento de $30 \mathrm{~mA}$. As medidas foram realizadas com o passo de $0,02^{\circ}$ e em tempo de 0,6

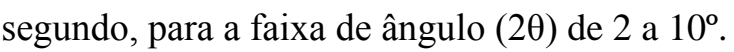

A análise de fluorescência de raios X por energia dispersiva (EDX) dos catalisadores sintetizados foi realizada em espectrômetro Shimadzu modelo 720. Os resultados obtidos foram expressos em teores de óxido de forma semi-quantitativa.

As microscopias eletrônicas de varredura foram obtidas em aparelho Philips, modelo XL30, com recobrimento de ouro nas amostras e realizada no Laboratório de Caracterização de Materiais-UFCG. As microscopias foram realizadas com ampliações de 2000x e 5000x.

\section{Resultados e Discussão}

\subsection{Difração de raios $X$}

As curvas de difração do suporte do suporte MCM-41 não calcinado e calcinado seguem representadas no Gráfico 1.

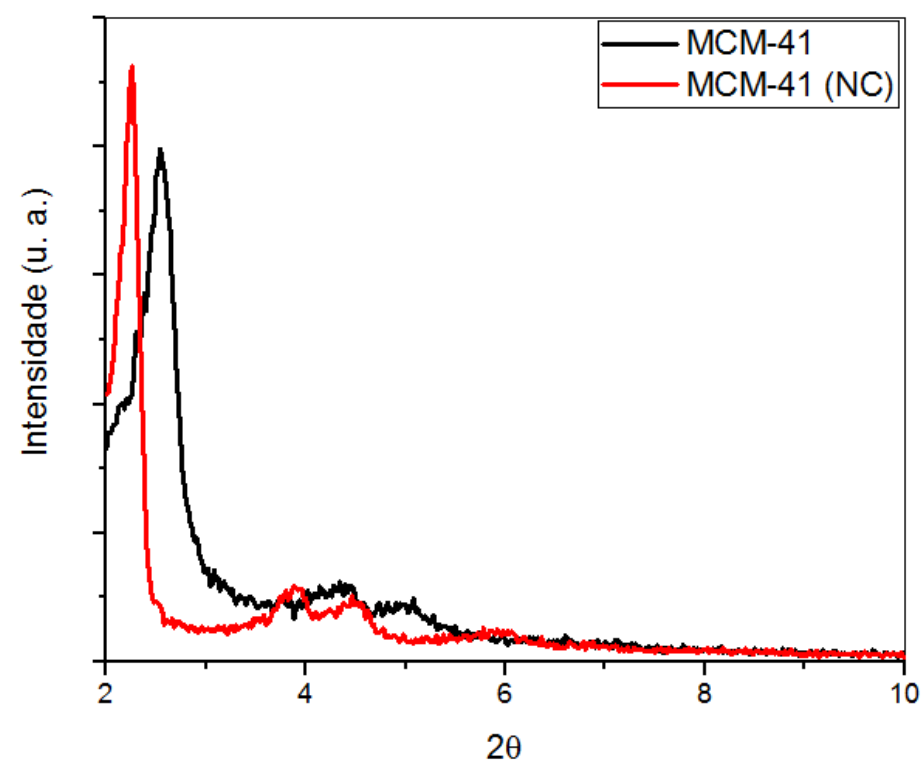

Gráfico 1 - Curva de difração de raios X para a MCM-41 calcinada e não calcinada.

Segundo Beck et al. (1992), a curva de difração padrão do MCM-41 apresenta de três a cinco picos de reflexão dos planos (100), (110), (200), (210) e (300). A presença de um único pico de reflexão do plano (100) já evidencia a formação da estrutura mesoporosa, porém de forma desordenada. Conforme constatado no Gráfico 1, a curva de difração da MCM-41 apresenta três picos característicos, indicando assim a formação da estrutura hexagonal característica da peneira molecular mesoporosa do tipo MCM-41.

Além disso, a caracterização realizada encontra-se em conformidade com os resultados da literatura obtidos por Gaydhankar et al. (2007), Antunes Júnior (2012a e 2012b) e Silva (2011), os quais verificaram a presença dos três picos de reflexão na curva de difração da MCM-41 sintetizada a temperatura ambiente e com mesma composição molar. 
Pela análise do Gráfico 1, observa-se que a etapa de calcinação promoveu uma redução da cristalinidade do material. A redução da intensidade do pico no plano 100 sugere uma menor ordenação das amostras após a etapa de tratamento térmico. Essa redução, no entanto, não promoveu a destruição do material e manteve as condições características do suporte mesoporoso. 2.

As curvas de difração para os catalisadores sintetizados seguem representadas no Gráfico

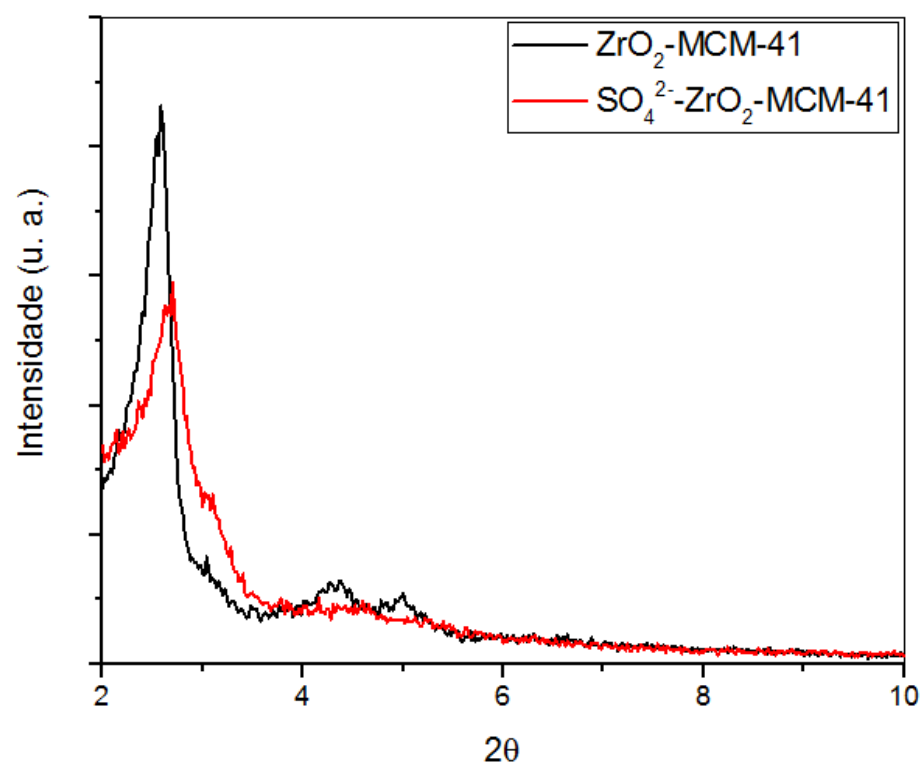

Gráfico 2 - Curva de difração de raios X para $\mathrm{ZrO}_{2}-\mathrm{MCM}-41$ e $\mathrm{SO}_{4}{ }^{2-}-\mathrm{ZrO}_{2}-\mathrm{MCM}-41$.

A partir da análise dos resultados contidos no Gráfico 2, verifica-se que o processo de incorporação do dióxido de zircônia não comprometeu severamente a estrutura no suporte mesoporoso. Além disso, verifica-se que houve redução da intensidade por conta da inserção de heteroátomos na estrutura do suporte.

Adicionalmente, observa-se que após a etapa de sulfatação do material ocorreu uma redução da intensidade dos picos característicos em relação ao catalisador com a incorporação da zircônia, assim como observado nos estudos de Ghedini et al. (2008), Chen et al. (2001) e Li et al. (2005).

Segundo Ghedini et al. (2008) a diminuição da intensidade dos picos característicos do material refere-se a um rearranjo desordenado da estrutura mesoporosa promovida pela sulfatação do catalisador sintetizado.

\subsection{Fluorescência de raios $X$ por energia dispersiva}

Os resultados obtidos por fluorescência de raios X por energia dispersiva (EDX) para o suporte calcinado, MCM-41, e para os catalisadores, $\mathrm{ZrO}_{2}-\mathrm{MCM}-41$ e $\mathrm{SO}_{4}{ }^{2-}-\mathrm{ZrO}_{2}-\mathrm{MCM}$ - encontram-se na Tabela 1.

Tabela 1 - Análise semi-quantitativa da composição do suporte e catalisadores.

\begin{tabular}{ccccc}
\hline Amostra & $\mathbf{S i O}_{\mathbf{2}} \mathbf{( \% )}$ & $\mathrm{ZrO}_{\mathbf{2}} \mathbf{( \% )}$ & $\mathbf{S O}_{\mathbf{3}} \mathbf{( \% )}$ & Outros (\%) \\
\hline MCM-41 & 96,713 & - & - & 3,287 \\
$\mathrm{ZrO}_{2}-\mathrm{MCM}-41$ & 91,395 & 4.645 & - & 3,960 \\
$\mathrm{SO}_{4}{ }^{2-}-\mathrm{ZrO}_{2}-\mathrm{MCM}-41$ & 92,110 & 3,156 & 2,136 & 2,598 \\
\hline
\end{tabular}


A partir dos resultados obtidos, observou-se que os suportes e catalisadores sintetizados são constituídos basicamente de óxido de silício. Além disso, verificou-se a presença do dióxido de zircônio $\left(\mathrm{ZrO}_{2}\right)$ e óxido derivado de enxofre nos catalisadores sintetizados.

A análise dos resultados obtidos através da caracterização por EDX demonstra que a etapa de incorporação do dióxido de zircônio pela dispersão física não foi totalmente eficiente por apresentar valores abaixo do valor teórico, porém próximos destes.

Após a etapa de sulfatação, no entanto, observa-se uma discrepância maior dos resultados referentes à concentração do óxido metálico indicando assim que esta etapa diminuiu a proporção do óxido pela análise semi-qualitativa. A este fato pode-se atribuir à etapa tratamento térmico, que não foi suficiente para uma completa difusão do dióxido de zircônio na superfície do catalisador. A etapa de calcinação utilizada constou de uma temperatura final de $550{ }^{\circ} \mathrm{C}$ por um tempo de $4 \mathrm{~h}$, enquanto que estudos de Wang e Mou (2008) adotaram temperatura de $720{ }^{\circ} \mathrm{C}$ e estudos de Chen et al. (2001a) utilizaram temperatura de $680^{\circ} \mathrm{C}$ por 3h. Além disso, estudos de Chen et al. (2001b) utilizaram a temperatura de $560{ }^{\circ} \mathrm{C}$ por um tempo de $6 \mathrm{~h}$ na calcinação do catalisador.

A concentração de outras substâncias nos suportes e catalisadores analisados indicou a presença de impurezas presentes nos reagentes utilizados para a síntese ou possível contaminação durante o procedimento de análise da caracterização.

\subsection{Microscopia Eletrônica de Varredura (MEV)}

Nas Figuras 1 a 3 encontram-se as micrografias eletrônicas de varredura obtidas para o suporte e catalisadores sintetizados.

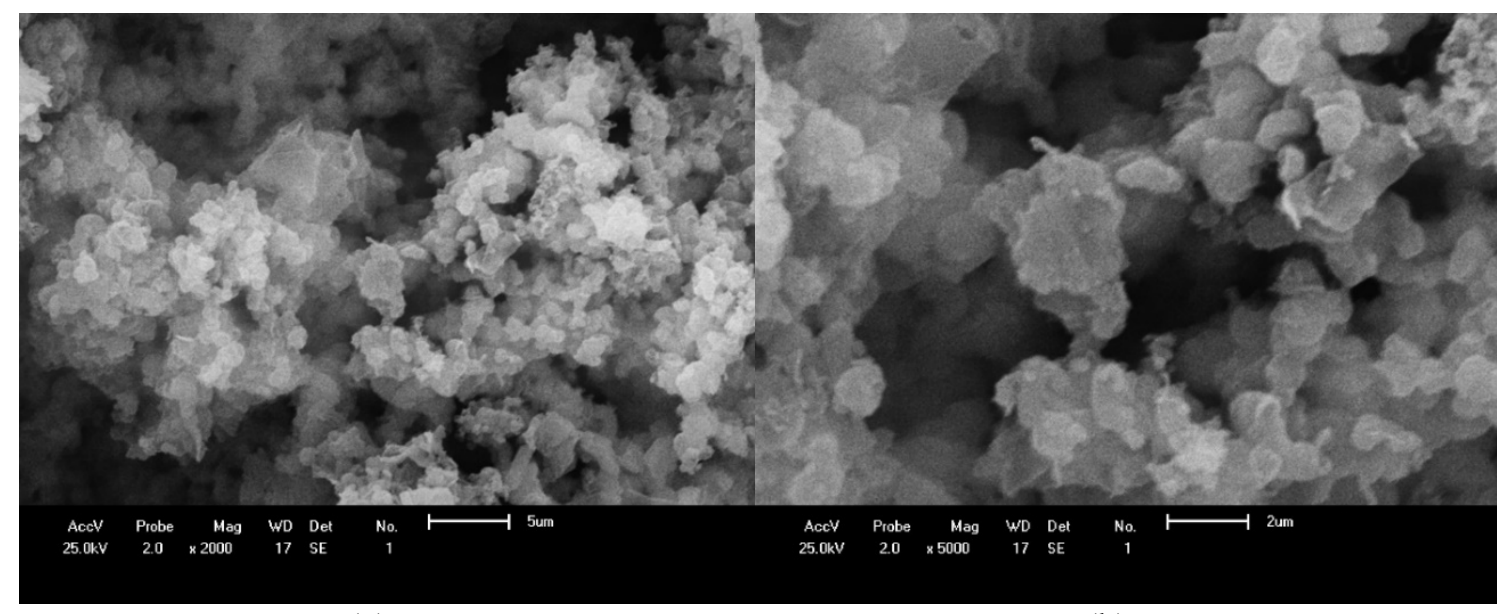

(a)

(b)

Figura 1 - Microscopia eletrônica de varredura do suporte MCM-41 com ampliação (a) 2000x e (b) 5000x. 


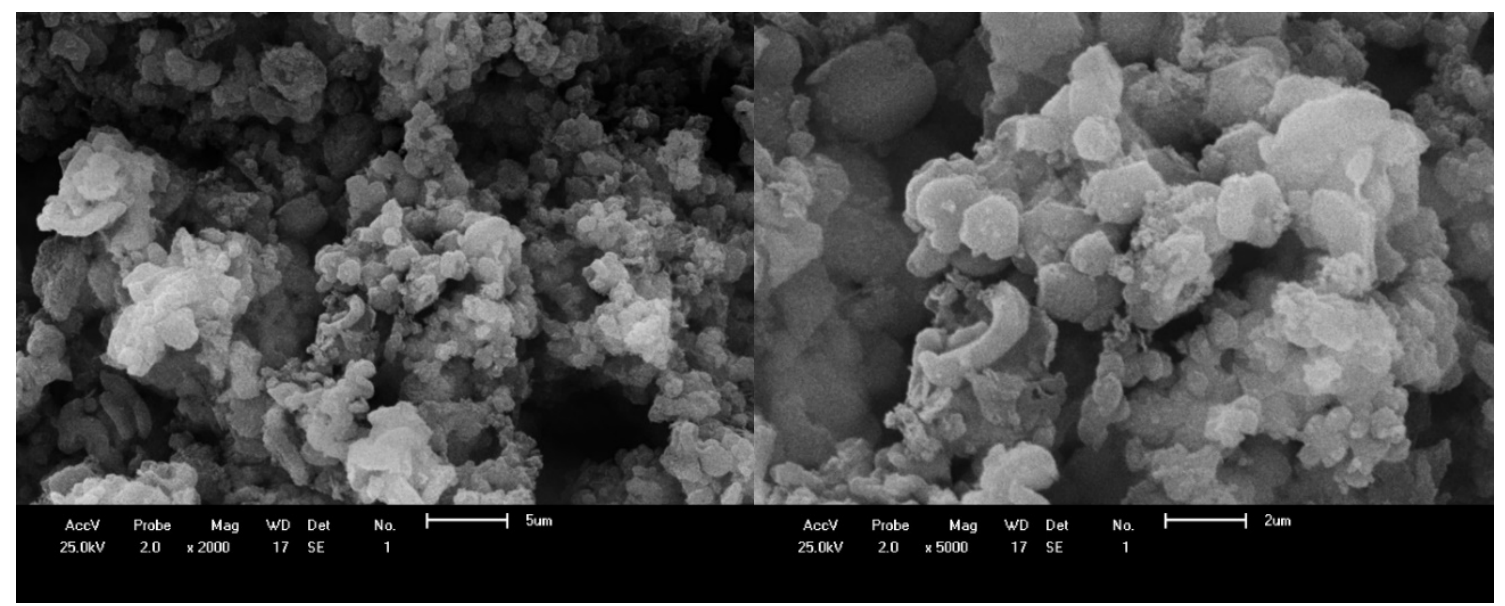

(a)

(b)

Figura 2 - Microscopia eletrônica de varredura do catalisador $\mathrm{ZrO}_{2}-\mathrm{MCM}-41$ com ampliação (a) 2000x e (b) 5000x.

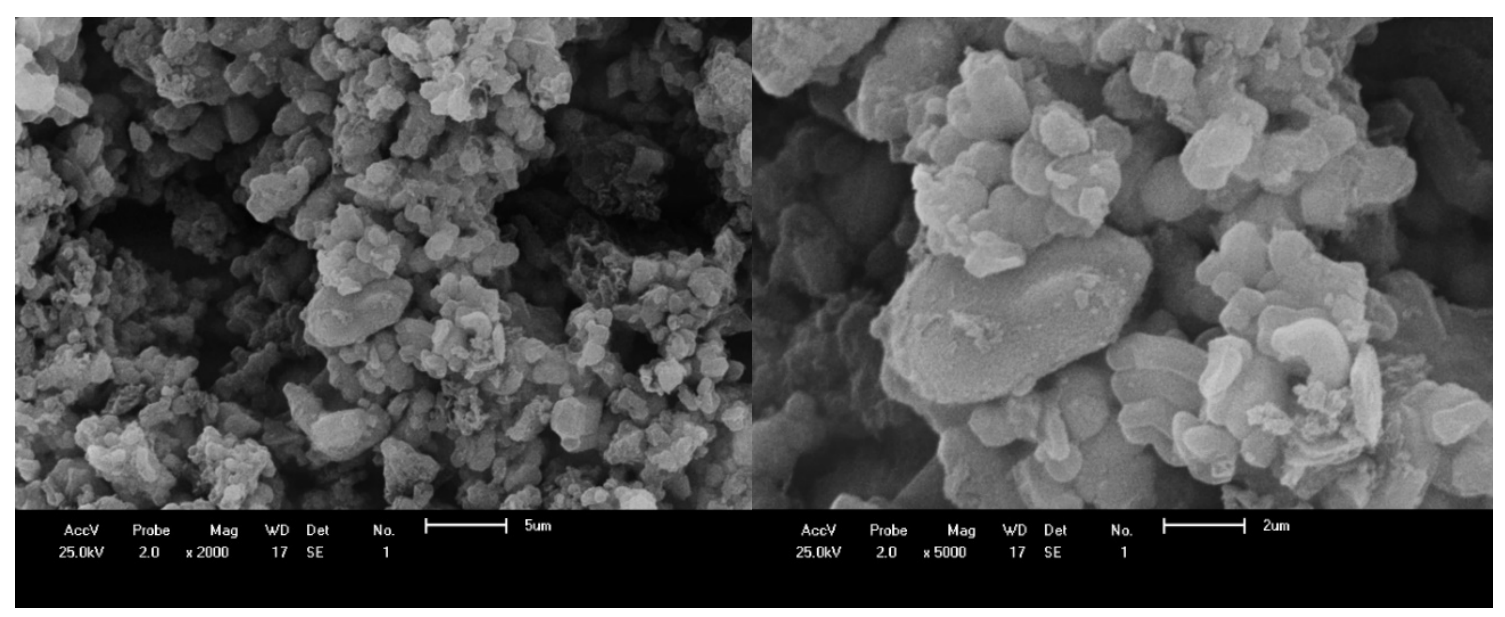

(a)

(b)

Figura 3 - Microscopia eletrônica de varredura do catalisador $\mathrm{SO}_{4}{ }^{2-}-\mathrm{ZrO}_{2}-\mathrm{MCM}-41 \mathrm{com}$ ampliação 2000x e (b) 5000x.

Através da análise das micrografias dos materiais sintetizados percebe-se que a morfologia do material varia bastante e não se pode distinguir precisamente o formato das partículas através destas ampliações. Tal fato poderia ser observado numa análise mais precisa ou com ampliações maiores. Apesar de forma indefinida, pode-se destacar a presença de algumas partículas com morfologia arredondada e bastões.

Em relação à dimensão das partículas nas amostras estudadas, verificou-se que o suporte apresentou aglomerados com tamanho variável entre 1 e $4 \mu \mathrm{m}$, quanto a amostra $\mathrm{ZrO}_{2}$-MCM-41 as partículas variaram entre 0,5 e $4 \mu \mathrm{m}$, enquanto que na amostra $\mathrm{SO}_{4}{ }^{2-}-\mathrm{ZrO}_{2}-\mathrm{MCM}-41$ as dimensões foram de 1 a $6 \mu \mathrm{m}$.

Além disso, observa-se a presença de aglomerados e partículas assimétricas após a etapa de incorporação do dióxido de zircônio e que o mesmo comportamento é verificado após a etapa de sulfatação do material. 


\section{Conclusão}

Com base nos resultados obtidos pode-se concluir que o processo de síntese a temperatura ambiente permite a obtenção da fase hexagonal mesoporosa da MCM-41. A incorporação do dióxido de zircônio apresentou valores próximos aos teóricos, porém após a etapa de sulfatação ocorreu uma maior disparidade entre estes valores, devido a introdução de novos compostos no material e pela técnica de EDX ser semi-qualitativa. A morfologia do material apresenta aglomerados de partículas sem forma bem definida.

O suporte mesoporoso MCM-41 e catalisadores sintetizados configuram-se como materiais promissores para aplicação catalítica em diversas áreas de processos químicos.

\section{Synthesis and characterization of sulfated MCM-41 materials}

\section{Albstract:}

Chemical processes consist on the transformation of raw materials into products through chemical reactions. Reactions in industrial processes need to have high efficiency, fast processing and good quality, which can be achieved through the use of catalysts. Due to environmental problems, catalysis shows up as a promisingalternativetopromotesustainablewaysofproductionandappears as fundamental to the improvement of chemical processes.

In general, the catalytic reactions can be classified into homogeneous and heterogeneous. The use of heterogeneous catalysts is highlighted because of the possibility of reducing environmental impacts and increasing process efficiency. Aiming to replacing homogeneous catalysts, the use of solid catalysts has been increasingly widespread in scientific circles. Mesoporous heterogeneous catalysts, such as Mobil Composition of Matter-41 (MCM-41), has been studied by many researchers in the application in reactions involving macromolecules. The main advantages of using this mesoporous molecular sieve are: high surface area, high pore diameter and pore volume and good thermal stability.

MCM-41 is catalytically inactive material or may present very weak acid sites, these mesoporous materials, in turn may have their surface modified by incorporating an active phase. Among which stand out the addition of heteroatoms to generate acid sites and the sulfate ion which gives the material the characteristic of superacid. Thus, the aim of this study was to evaluate the synthesis and characterization of MCM-41 support and $\mathrm{ZrO}_{2}-\mathrm{MCM}-41$ and $\mathrm{SO}_{4}{ }^{2-}-$ $\mathrm{ZrO}_{2}$-MCM-41 catalysts. The catalysts were synthesized by incorporation physical mixture method with a concentration of $5 \%$ in mass ratio of $\mathrm{ZrO}_{2}$ and then characterized by XRD, SEM and EDX.

The results suggest that the behavior characteristic of mesoporous materials of the MCM41 was reached after synthesis and the materials are essentially constituted by silica. Alternatively, it was observed the presence of zirconia and sulfur based compounds after analysis of catalysts. In relation to morphology, it was found that the materials exhibit heterogeneous forms with the presence of agglomerates.

Keywords: Molecular sieves; MCM-41; adsorption; Heterogeneous catalysis.

\section{Referências bibliográficas}


ANTUNES JÚNIOR, A. U.; PEREIRA, K. R. O.; SILVA, A. S.; CARVALHO, M. W. N. C., Síntese de catalisadores do tipo MCM-41 e Al-MCM-41 para aplicação em reação de esterificação de óleo de soja, in: IX Encontro Brasileiro sobre Adsorção \&I Simpósio Ibero-Americano sobre Adsorção. Recife, 2012 (a).

ANTUNES JÚNIOR, A. U.; LEITE, C. E. T.; SANTOS, T. S. S.; SILVA, A. S.; PEREIRA, K. R. O.; CARVALHO, M. W. N. C., Avaliação da influência de Fe, Ni e Mo impregnado em MCM-41 para produção de biodiesel, in: XIX Congresso Brasileiro de Engenharia Química. Búzios, 2012 (b).

ARMOR, J. N., A history of industrial catalysis, Catalysis Today, v. 163, p. 3-9, 2011.

AQUINO, J. M. F. B.; SOUZA, C. D. R.; ARAÚJO, A. S. Synthesis and characterization of sulfatesupported MCM-41 material. International Journal of Inorganic Materials, v. 3, p. 467-470, 2001.

BECK, J. S.; VARTULI, J. C.; ROTH, W. J.; LEONOWICZ, M. E.; KRESGE, C. T.; SCHMITT, K. D.; CHU, C. T. W.; OLSON, D. H.; SHEPPARD, E. W.; A new family of mesoporous molecular sieves prepared with liquid crystal templating. Journal American Chemical Society, v.114, p.10834, 1992.

CASTRO, K. K. V. Síntese, caracterização e aplicação do MCM-41 e Al-MCM-41 na pirólise do resíduo atmosférico de petróleo, Dissertação de Mestrado, Universidade Federal do Rio Grande do Norte. Natal, 2009.

CHEN, C. L.; LI, T.; CHENG, S.; LIN, H.P.; BHONGALE, C. J.; MOU, C. Y., Direct impregnation method for preparing sulfated zirconia supported on mesoporous silica, Microporous and Mesoporous Materials, v. 50, p.201-208, 2001a.

CHEN, C. L.; CHENG, S.; LIN, H. P.; WONG, S. T.; MOU, C. Y., Sulfated zirconia catalyst supported on MCM-41mesoporous molecular sieve, Applied Catalysis A: General, v. 215, p. 21-30, 2001b.

CHEN, Y.; CHEN, X.; DONG, B.; WANG, G.; ZHENG, X., Facile Synthesis and Characterization of 12Tungstophosphoric Acid Anchoring MCM-41 Mesoporous Materials, Materials Letters, v. 114, p. 72-75, 2014.

CORMA, A., From microporous to mesoporous molecular sieve materials and their use in catalysis, Chemical Review, v. 97, p. 2373, 1997.

GAYDHANKAR, T. R.; SAMUEL, V.; JHA, R. K.; KUMAR, R.; JOSHI, P. N. Room temperature synthesis of Si-MCM-41 using polymeric version of ethyl silicate as a source of silica. Materials Research Bulletin, v. 42, p. 1473-1484, 2007.

GHEDENI, E.; SIGNORETTO, M.; PINNA, F.; CRUCIANI, G.; Mesoporous Silica-Zirconia Systems for Catalytic Applications, Catalysis Letters, v. 125, p. 359-370, 2008.

LI, L.; YU, S.; LIU, F.; YANG, J.; ZHAUG, S., Reactions of turpentine using Zr-MCM-41 family mesoporous molecular sieves, Catalysis Letters, v. 100, p. 227-233, 2005.

MORENO, E. L.; RAJAGOPAL, K., Desafios da acidez na catálise em estado sólido, Química Nova, v. 32, p. 538-542, 2009.

ONDA, A.; OCHI, T.; YANAGISAWA, K. Hydrolysis of cellulose selectively into glucose over sulfonated activated-carbon catalyst under hydrothermal conditions. Topics in Catalysis, v. 52, p. 801-807, 2009.

PARVULESCU, V.; CMAN, S.; PARVULESCU, V. I.; GRANGE, P.; PONCELET, G. Reaction of hexane, cyclohexane, and methylcyclopentane over gallium-, indium-, and thallium-promoted sulfated zirconia, Journal of Catalysis, v.180, p.66-84, 1998.

SCHMAL, M., Catálise heterogênea, 1a ed., Synergia. Rio de Janeiro, 2011. 
SILVA, A. S., Avaliação de catalisadores de $\mathrm{NiO}$ e $\mathrm{MoO}_{3}$, suportados em MCM-41, na obtenção de biodiesel de óleo de algodão, Tese (Doutorado em Engenharia de Processos), Universidade Federal de Campina Grande. Campina Grande, 2011.

SU, F.; GUO, Y., Advancements in solid acid catalysts for biodiesel production, Green Chemistry, v. 16, p. 2934-2957, 2014.

SUN, Y.; ZHU, L.; LU, H.; WANG,E.; LIN, S.; JIANG, D.; XIAO F. Sulfated zirconia supported in mesoporous materials. Applied Catalysis A: General, v. 237, p.21-31, 2002.

TANABE, K.; HÖLDERICH, W. F. Industrial application of solid acid-basecatalysts, Applied Catalysis A: General, v. 181, p. 399-434, 1999.

VÉDRINE, J. C., Revisiting active sites in heterogeneous catalysis: their structure and their dynamic behaviour, Applied Catalysis A: General, v. 474, p. 40-50, 2014.

WANG, J. H.; MOU, C. Y., Characterizations of aluminum-promoted sulfated zirconia on mesoporous MCM-41 silica: Butane isomerization, Microporous and Mesoporous Materials, $\mathrm{v}$. 110 , p. 260-270, 2008.

YANG, G.; DENG, Y.; WANG, J., Non-Hydrothermal Synthesis and Characterization of MCM-41 Mesoporous Materials from Iron Ore Tailing, Ceramics International, v. 40, p. 7401-7406, 2014.

ZHANG, J.; CHEN, S.; YANG, R.; YAN, Y., Biodiesel production from vegetable oil using heterogeneous acid and alkali catalyst, Fuel, v. 89, p. 2939-2944, 2010. 